\title{
Adaptaciones en la arquitectura del Centro Histórico de Granada, Nicaragua
}

\author{
Architectural Adaptation in The Historical Centre of Granada, Nicaragua
}

\author{
Luis Carlos, Cruz-Ramirez ${ }^{1}$ \\ ${ }^{1}$ Instituto Politécnico Nacional, ESIA-Tecamachalco, Estado de México \\ luisram06@gmail.com / https://orcid.org/0000-0002-3651-4471 \\ Recibido el 16 de septiembre de 2021, aprobado el 14 de diciembre de 2021
}

\begin{abstract}
RESUMEN | En este artículo se explica la relación entre la dotación de equipamiento turístico y la adaptación de la forma de los edificios en los centros históricos. Se seleccionó como objeto de estudio el centro histórico de la ciudad de Granada, Nicaragua. De todos los géneros de edificios, se estudió la dotación en edificios habitacionales. Posteriormente, se tomó una muestra de edificios que experimentaron cambio de uso, de habitacional a equipamiento turístico. Se analizaron los espacios que cambiaron su uso, así como las posibles adaptaciones. Estos datos se ordenaron y se les asignaron valores para posteriormente realizar análisis probabilísticos. Se encontró que existe una mayor frecuencia (63.2\%) de espacios que experimentaron adaptaciones formales cuando existe dotación turística. Existe una relación entre el cambio de uso de los espacios y las adaptaciones, con correlación de 77.21\%. Estos resultados indican que, a mayor cantidad de cambios de usos en un edificio, se presenta mayor cantidad de modificaciones formales. Estas modificaciones se tienden a llevar a cabo para que los espacios funcionen. Se deben principalmente a que las actividades turísticas desarrolladas en los espacios arquitectónicos son diferentes a las actividades realizadas en la vivienda. Estos hallazgos pueden ser de utilidad para la orientación de los proyectos de intervención en edificios patrimoniales.
\end{abstract}

PALABRAS CLAVE | Adaptaciones, arquitectura, centro histórico, dotación turística, modificaciones

ABSTRACT | In this paper, association between the provision of tourist equipment and modification of the shape of buildings in historic centres is explained. To develop the aforementioned relation, the historic centre of the city of Granada, Nicaragua was selected as the object of study. Among all gender of buildings, endowment in residential buildings is studied. Subsequently, a sample of buildings that underwent change of use, from housing to tourist facilities, was taken. Architectural spaces that changed their use were analysed, as well as their possible adaptations. These data were ordered and some values were assigned them to later perform probabilistic analyses. It was found that, there is a higher frequency $(63.2 \%)$ of spaces that underwent formal adaptations when there are tourist provisions. There is a relationship between the change in use of spaces and adaptations, a correlation equal to $77.21 \%$. Results indicates that, when the number of changes in uses in a building increase, also the number 
of formal modifications rise. This fact is mainly due to tourist activities carried out in the architectural spaces are different from the activities implemented in dwelling. These findings can be useful to guide intervention projects in heritage buildings.

KEYWORD | Adaptation, architecture, historical centre, modifications, touristic endowment

\section{Introducción}

El objetivo principal de este artículo es explicar la relación que existe entre la dotación de equipamiento turístico y la modificación de la forma de los edificios en los centros históricos. Los centros históricos son una región en la ciudad, que se caracterizan principalmente por contener una estructura heredada del pasado (UNESCO, 1977, p. 13). Esto incluye su arquitectura, que al igual que el resto de la ciudad -incluyendo los sectores intermedios y periféricos (Mattos, 2002)-, experimenta procesos de adaptación por acción del ser humano (Ellisa, Narumi y Tahaka, 1997).

Una adaptación, conceptualmente, es un proceso en el que se ajustan los sistemas naturales o humanos, los cuales sirven para moderar los posibles daños y aprovecha las oportunidades o beneficios (Köspsel, 2019, p. 23). En la arquitectura, esto se puede interpretar como el ajuste de la obra arquitectónica para obtener el mayor beneficio o aprovechar los espacios arquitectónicos existentes, según las necesidades. Dicho ajuste consiste en un cambio intencional de una o más característica de un objeto con el fin de obtener un beneficio. Con base en lo anterior, la adaptación puede presentarse en la forma, apariencia o el uso del edificio. Por ejemplo, el término reúso adaptable es el proceso de conversión del edificio ajustado a nuevos requisitos funcionales (Eyüce \& Eyüce, 2010, p. 419).

La adaptación formal arquitectónica es un fenómeno fácilmente perceptible. Se pueden observar al realizar una serie de visitas a los centros históricos y establecer una relación entre las características del pasado y del presente. Uno de los cambios que tienden a ocurrir en los centros históricos que son frecuentemente visitados, es que los edificios que eran habitacionales ahora son hoteles, hospedajes, restaurantes, o tiendas de souvenir. Suele ocurrir que estos cambios de uso en los edificios, llevan consigo adaptaciones formales. Así es como las casas que antes constaban de un sólo nivel, ahora tienen dos o más niveles; otras han sido divididas, dando origen a dos o más edificios. Los espacios de las viviendas ahora están divididos, produciendo otras configuraciones espaciales en la arquitectura. La proporción geométrica interior de los espacios ha cambiado, junto con las medidas de anchura y longitud, esto sucede con la adición o sustracción de muros. Las paredes exteriores pueden presentar mayor cantidad de vanos que los existentes previamente. Por ejemplo, si en el pasado la puerta de acceso era el único vano, ahora tienen una serie de ventanas o más puertas de acceso. La descripción anterior corresponde al hecho que se denominará en este estudio como la adaptación de forma en los edificios habitacionales de los centros históricos.

Pero ¿Qué es lo que está generando este fenómeno? Estos hechos observados pueden estar asociados a diversos tipos de agentes de cambio. Uno de estos, es la dotación de equipamiento turístico (Suebsuk \& Nakagawa, 2015; Ellisa, Narumi, \& Tahara, 1997; Soewarno \& Duhita, 2019). Para contextualizar, el turismo consiste en el desplazamiento voluntario y temporal por motivos de recreación, descanso, cultura o salud, en el cual las personas no ejercen ninguna actividad lucrativa ni remunerada, generando interrelaciones sociales, económicas y culturales (De la Torre, 1998, p. 16). Esta actividad puede ser realizada por uno o más individuos sin participar en los mercados de 
trabajo y capital de los sitios visitados (Hernández, 1991, p. 12) de un atractivo turístico.

En los estudios de turismo, los centros históricos y su arquitectura, se les considera un atractivo turístico (Speach, 2014, pp. 24-40). Esto porque son un tipo de agente que incentiva a las personas a desplazarse voluntariamente (Boullón, 1985, p. 45), por el interés de conocer construcciones históricas y patrimoniales. Internacionalmente, los centros históricos están catalogados en la subcategoría de lugares históricos y sus edificios, incluidos en el grupo de arquitectura popular o vernácula, dentro de los atractivos folclóricos (De la Torre Padilla, 1997). Además, entre la variedad de edificios, un centro histórico puede contener museos, reconstrucciones, arqueología industrial, fortalezas y, catedrales, construcciones consideradas atractivos del turismo patrimonial (Robinson, 2012, pp. 95-99).

Conceptualmente, la dotación de bienes y servicios, es la acción de proporcionar o suministrar servicios a la comunidad (Camacho, 1993, p. 273). En este contexto, la dotación de equipamiento turístico en centros históricos, es la acción de proporcionar edificios de equipamiento a la ciudad histórica, para que el turista lo utilice y pueda cumplir sus necesidades de alojamiento, alimentación y recreación.

Pero, ¿Cómo es que la dotación turística influye en las adaptaciones de la arquitectura habitacional del centro histórico? La hipótesis que se sostiene es que las dotaciones de usos turísticos, representan por sí mismas cambios de uso de los espacio -porque el nuevo uso del edificio implica el proceso de conversión arquitectónico para ajustarse a nuevos requisitos funcionales en dependencia de las nuevas necesidades-. Estos cambios en el uso traen consigo adaptaciones formales para poder desempeñar actividades de una manera confortable. Sin embargo, las adaptaciones formarles estarán en dependencia de la relación que exista entre el uso previo y el uso actual, en la medida que estos usos difieran más, existirá mayor probabilidad de encontrar un espacio adaptado formalmente.

Para dar respuesta a esta pregunta y explicar la relación antes mencionada, se seleccionó como objeto de estudio el centro histórico de la ciudad de Granada, Nicaragua (ver Figura 1). De todos los géneros de edificios, se estudia la dotación en edificios habitacionales. La arquitectura habitacional, es el género de edificios predominante (Oliver, 2006, p. xxi), por lo que también es el que experimenta mayor frecuencia de cambios, debido a que las personas tienden a realizar modificaciones para adáptalos a sus necesidades (Suebsuk \& Nakagawa, 2015). Posteriormente, se tomó una muestra de edificios que experimentaron cambio de uso, de habitacional a equipamiento turístico. Se analizaron los espacios que cambiaron su uso, así como las posibles transformaciones. Estos datos se ordenaron y se les asignaron valores para posteriormente realizar análisis probabilísticos. La explicación de este problema puede ser de utilidad para regular la acción turística en los centros históricos, asimismo, puede ser útil para orientar las intervenciones en edificios patrimoniales y evitar daños irrecuperables.

Este artículo se desarrolla en tres secciones: primero se presenta una sección teórica, donde se explica como ocurre el hecho de la modificación de la forma por la dotación de equipamiento turístico. Proceso que inicia con la formación de la ciudad turística, pasa por la interacción entre la oferta y la demanda turística, y culmina en los modos de ocupación del espacio y sus posibles adecuaciones. Luego se presenta una sección de método donde se explica la manera en que se realizó la prueba, considerando el objeto de estudio, la toma de datos y el análisis de los mismos. Posteriormente, se presentan los principales resultados encontrados. Finalmente, algunos comentarios finales con sus implicaciones. 
Figura 2. Adaptación turística de la calle peatonal La Calzada, Centro histórico de Granada, Nicaragua. Foto del autor.

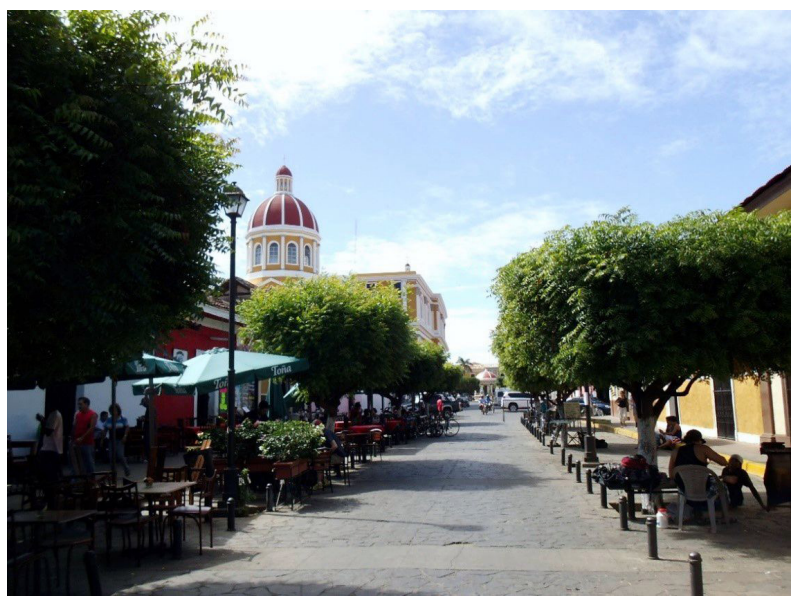

Nota. Foto del autor

\section{Modificaciones formales por dotación de equipamiento}

Para que ocurra la dotación de equipamiento turístico, previamente existe un proceso de conversión de la ciudad histórica al modelo turístico. En este proceso intervienen los habitantes locales del centro histórico, los turistas, las políticas y los edificios. Primero, se conforma la ciudad histórica, luego se produce una interacción entre la oferta y la demanda de servicios a causa de las necesidades de la población visitante en aumento, y finalmente, la adecuación de los edificios para ofertar los servicios. Este proceso se explica a continuación.

\section{Formación de la ciudad histórica}

El proceso de formación de la ciudad histórica ocurre en tres fases (Ashworth, 2005). En la primera fase, denominada formación de la ciudad originaria, es cuando se genera el asentamiento humano, este crece y adquiere funciones urbanas hasta ser una ciudad (p. 66). En la segunda fase, la ciudad continúa creciendo y se convierte en histórica -cuando crece hacia el exterior-, es así como el centro que fue toda la ciudad en la primera etapa adquiere valor histórico, porque es lo más antiguo de la ciudad (p. 66). Finalmente, en la tercera fase, ocurre una revalorización del patrimonio histórico, que permite la introducción de políticas de preservación y conservación, que generalmente concierne a la relación entre la ciudad histórica y la función comercial central de la ciudad (p. 66).

\section{La oferta y la demanda turística}

Este proceso inicia cuando el turista visita el centro histórico, ya sea por la necesidad de conocer, por ocio o por entretenimiento. A estas necesidades se le dominan en este artículo como necesidades de visita. Estas necesidades de visita, son las que generan el desplazamiento inicial de los visitantes hacia la ciudad turística, dan origen a otras necesidades específicas relacionadas con la permanencia en el destino turístico. Entre las necesidades de los turistas se encuentran: desplazarse temporalmente, comer, hospedarse y distraerse. Dichas necesidades establecen una sobre demanda local y se suple, en parte, con la infraestructura existente.

Estas necesidades de los turistas están relacionadas con los tipos de servicios o recursos turísticos y son el origen de las ofertas (De la Torre, 1998, pp. 22-23). Existen dos tipos de recursos: los primarios son los que motivan la visita y los secundarios son los recursos de apoyo del visitante 
durante la estancia (Ashworth, 2005, p. 75). Los primarios son atractivos turísticos y los secundarios son los equipamientos.

Cuando un centro histórico es reconocido socialmente como un atractivo turístico, se produce un incremento de población visitante, que es temporal. Al igual que la población local, los visitantes tienen necesidades y requieren de servicios de equipamiento. Es así como cada servicio turístico ofertado en un centro histórico, puede estar asociado con alguna necesidad (Soewarno \& Duhita, 2019, p. 131), que tiende a crecer al aumentar la población visitante.

Al tratarse de un sitio consolidado, las edificaciones predominantes que en un inicio fueron habitacionales, luego son equipadas para ofertar servicios que no existen o no son suficientes (Suebsuk \& Nakagawa, 2015, p. 48). Por otro lado, desde el estudio del proceso de diseño arquitectónico, las necesidades dan origen a usos y ocupaciones espaciales (Groat \& Wang, 2012). Esta modificación de uso puede ser parciales o totales. Parciales cuando un nivel o una región del edificio es utilizada para realizar actividades para ofertar un servicio, mientras otra parte continúa siendo habitacional. El cambio es total cuando el edificio deja de ser habitacional. Este cambio de uso conlleva también al cambio o adaptación formal una vez que los edificios son ocupados para otros fines.

\section{Modos de ocupación y adecuaciones}

Las modificaciones formales que se realizan en los edificios dependen de las necesidades del sujeto, las cualidades del espacio, compatibilidad entre uso previo y el uso actual, y la interpretación del espacio por el usuario. La necesidad de desarrollar actividades relacionadas con la oferta turística de servicios cambia los modos de ocupación ${ }^{l}$ del espacio existente. Cuando se analiza este hecho por la vía del funcionalismo arquitectónico, cada edificio de acuerdo a su género tendrá su propio flujo funcional, es decir que, para su óptimo aprovechamiento, según el objetivo fijado, requiere el cumplimiento de un programa arquitectónico. Por tanto, la dotación de cada servicio tendrá una manera particular de funcionar y ésta se asocia con las formas necesarias para desarrollar esas actividades.

Cuando los espacios propuestos cumplen con las condiciones existentes en cuanto a dimensiones y configuración, el cambio de uso no requiere adaptaciones formales. Toda acción requiere de un espacio determinado, algunas veces este espacio debe estar medido con precisión, en otras ocasiones puede variar dentro de los límites determinados, es decir, que el espacio debe estar en el rango de lo mínimo necesario para realizar una acción y el máximo permisibles sin que pierda el carácter práctico (Norberg-Schulz, 2001, p. 74).

Cualquier nueva función comercial localizada en una centralidad puede ocupar un edificio ya existente, convertir este edificio o destruir su estructura original y crear una nueva forma (Davies, 1968, p. 91 citado en Carter, 1972/1974, pp. 364-365). El cambio de forma de los edificios puede ser producto de la variación de las acciones que se llevan a cabo en los interiores de los espacios, que se debe a la necesidad de resolver el problema de permanencia del ser humano en un espacio por un tiempo limitado. El modelo propuesto por Davies (1968) consiste en estudiar las formas de los edificios en dos etapas: previa y actual. Considera los tipos de ocupación del edificio y la función necesaria en relación con la forma preexistente (Carter, 1972/1974, pp. 364-365).

También es necesario establecer una relación entre el uso previo y el uso turístico propuesto. Al comparar el uso previo con uso actual, se pueden encontrar tres variantes: el mismo uso, uso

1 Este término se implementa en este artículo para indicar la manera particular en que el sujeto utiliza el espacio arquitectónico E-ISSN: 2518-2943 | WEB: www.camjol.info/index.php/arquitectura | DOI: https://doi.org/10.5377/arquitectura.v6i12.13096 | E-MAIL: rvarqui.mas@farq.uni.edu.ni 
similar o uso diferente (Flores, 1999, p. 57). Cuando es el mismo uso, no se requiere de cambios ni modificaciones (p. 57). Por otro lado, si el uso es similar, las adaptaciones que deben hacerse al edificio para hacer factible la propuesta, no requieren de modificaciones en cuanto al espacio construido (p. 57). Sin embargo, cuando el uso es diferente, las necesidades de espacio entre la disposición original del edificio y la propuesta de uso, se requiere de modificaciones (p. 57). Con base en las afirmaciones anteriores, las modificaciones dependerán de las condiciones de los espacios existentes en las edificaciones habitacionales, por lo que será necesario adaptar y transformar aquellos edificios para que sean funcionales, cuando la variación de funciones tienda a ser más distante.

Lo anterior significa que, al tratarse de edificios habitacionales que cambian a servicio de alojamiento, en términos generales podría adaptarse por la similitud de uso de sus espacios, pero los cambios estarían dirigidos a la oferta de comodidades a los clientes que pernoctan, así como los servicios extras que se oferten que podría generar la adición de volúmenes. Por otro lado, los servicios de alimentación requieren de espacios sin divisiones para la localización del área de comensales, que podría generar la unión de espacios, pero se podrían adaptar al dividir a los comensales por tipo de servicio especializados que se oferten. Por tanto, la clase de servicio turístico a dotar puede generar tipos de modificaciones en la forma de la arquitectura existente.

Finalmente, el uso del espacio depende de la interpretación que haga el sujeto sobre el mismo espacio. Al estudiar la relación del sujeto con el objeto arquitectónico, sucede un acto de comunicación. El código arquitectónico produce un código icónico, donde el objeto se convierte en un elemento de comunicación (Eco, 1968/1986, p. 254). El sujeto por medio de la percepción genera una idea del edificio y el espacio, un concepto del objeto. La idea del objeto arquitectónico es una comunicación de la posible función (p. 254).

La comunicación es una estimulación; el estímulo es un complejo de actos sensoriales que provocan una determinada reacción (p. 255). Un espacio es un estímulo y por cada estimulo existe una conducta (p. 255). La conducta es la reacción al estímulo espacio, y según sea comprendido el espacio será el uso que realice el sujeto. Los estímulos son percibidos y comprendidos por la experiencia (p. 255), entonces, cuando el sujeto intenta utilizar un espacio arquitectónico existente, observa y asocia formas existentes y con sus maneras conocidas o posibles de aprovecharlas. Así, el objeto puede tener variedad de formas, pero el sujeto lo reconoce a partir de características abstractas del objeto codificado. Es decir, a partir de las características tipológicas. El uso de la arquitectura no solamente son las funciones posibles, sino los significados vinculados a estas, que predisponen la ocupación del espacio (p. 256). Cada sujeto tendrá una manera diferente de reaccionar al espacio, donde la reacción estará en función de la manera en que utiliza el edificio. Así el sujeto a cargo de la adaptación decide la utilización del espacio arquitectónico a partir de lo que percibe. De esta forma varía la manera en que puede utilizar el espacio una persona que conoce cómo funciona una actividad de servicio turístico, una profesional en la intervención de edificios históricos o alguien que lo desconoce.

Con base en las afirmaciones anteriores, la hipótesis es la siguiente: las dotaciones de usos turísticos, representan por sí mismas, cambios de uso de los espacios porque el nuevo uso del edificio necesita resolver otra categoría de necesidades. Estos cambios en el uso traen consigo adaptaciones formales para poder desempeñar actividades de una manera confortable, según lo que cada sujeto perciba del espacio y del conocimiento que tenga sobre la actividad. Sin embargo, las adaptaciones formarles estarán en dependencia de la relación que exista entre el uso previo y el uso actual, en la medida que estos usos difieran más, existirá mayor probabilidad de encontrar un 
espacio adaptado formalmente.

\section{Método}

Para comprobar la hipótesis, primero se delimitaron las variables de la investigación: (a) la dotación de equipamiento turístico y (b) la adaptación de los edificios. Los indicadores de la dotación de equipamiento corresponden a la existencia de los usos destinados a los turistas. Estos se clasificaron en cuatro grupos: alojamiento, alimentación, recreación y una cuarta categoría nombrada como otros ${ }^{2}$. Para el análisis de la adaptación de edificios, se tomó como indicador el cambio de configuración. Este indicador solamente se puede identificar por medio de un contraste entre la forma de la distribución previa y posterior a tomar la función de equipamiento turístico.

Luego se procedió a buscar locaciones donde estudiar el fenómeno de la dotación de equipamiento turístico en centros históricos. Para esto, se seleccionó el centro histórico de la ciudad Granada, Nicaragua, debido a que es un centro histórico con incidencia turística, por la factibilidad -económica y temporal- y por la practicidad de recorrerlo. Según Oficina de Preservación del Centro Histórico de Granada Nicaragua (OPCHCG, 2002, p. 3), el centro histórico de la ciudad de Granada fue definido por Alcaldía de Granada en 1982, este a su vez redefinido en 2002, por OPCHCG cuando aumentó notablemente la afluencia de turistas.

Los objetos de estudio son los edificios habitacionales con adaptaciones ubicados dentro del centro histórico seleccionado. Para poder tener una primera aproximación a la proporción de la dotación de equipamiento turístico e identificar los casos potenciales de estudio, primero se obtuvo un plano lotificado de la ciudad de Granada con sus límites. Con esta fuente de información, se realizó un recorrido por las calles -primarias y secundarias, peatonales y vehiculares- dentro de este centro histórico para registrar los usos de los edificios. Se elaboró una clasificación de los mismos, con base en las categorías de usos urbanos propuestos por Bazant (2005, pp. 156-159): uso habitacional o equipamiento. Entre los tipos de equipamiento se pueden mencionar: administración pública, cultural, religión, salud, asistencia social, abasto, administrativo, comunicaciones, recreación, infraestructura y servicios. Posteriormente estos se agruparon según los usos turísticos antes mencionados. Durante los recorridos, se logró registrar manualmente -con el auxilio de planos físicos-, el uso de 508 (54\%) edificios, de una población total representada por 928 edificios -identificadas previamente en un plano catastral-. De estos 508 edificios, 124 edificios tenían unos turísticos (ver Figura 2).

A partir de esta información, se procedió a la selección de los casos de estudio según los siguientes criterios de elegibilidad y exclusión. Se eligieron únicamente los edificios con registros de configuraciones documentados ${ }^{3}$-en forma planimétrica o fotográfica-, con uso habitacional y que experimentaron cambios de a uso turístico. Otro criterio de elegibilidad, fue la posibilidad de realizar recorridos en los interiores del edificio para poder identificar configuraciones actuales y poder contrastar la adaptación. Quedaron excluidos todos aquellos edificios, que tenían usos turísticos, previo a la identificación y redefinición del centro histórico. Se pudo encontrar con base en (a) una revisión bibliográfica de OPCHG $(2002,1999)$ y (b) preguntas a los pobladores locales, que tres de los 124 edificios de equipamiento de servicio turístico fueron construidos desde antes de la década de 1990 como hoteles de paso. Con esto se limitó a 121 edificios de equipamientos, que fueron casas de habitación antes de 2002 - momento en que se redelimitó el centro histórico

2 En la categoría de otros, se incluyeron las tiendas de recuerdos, oficinas informativas, servicios de transporte y guías turísticos. Esto se debe a que la cantidad de observaciones registradas eran relativamente la minoría.

3 En libros de textos, inventarios y publicaciones locales de propietarios de los edificios. 
de Granada-.

Figura 2. Edificios de equipamiento turísticos identificados

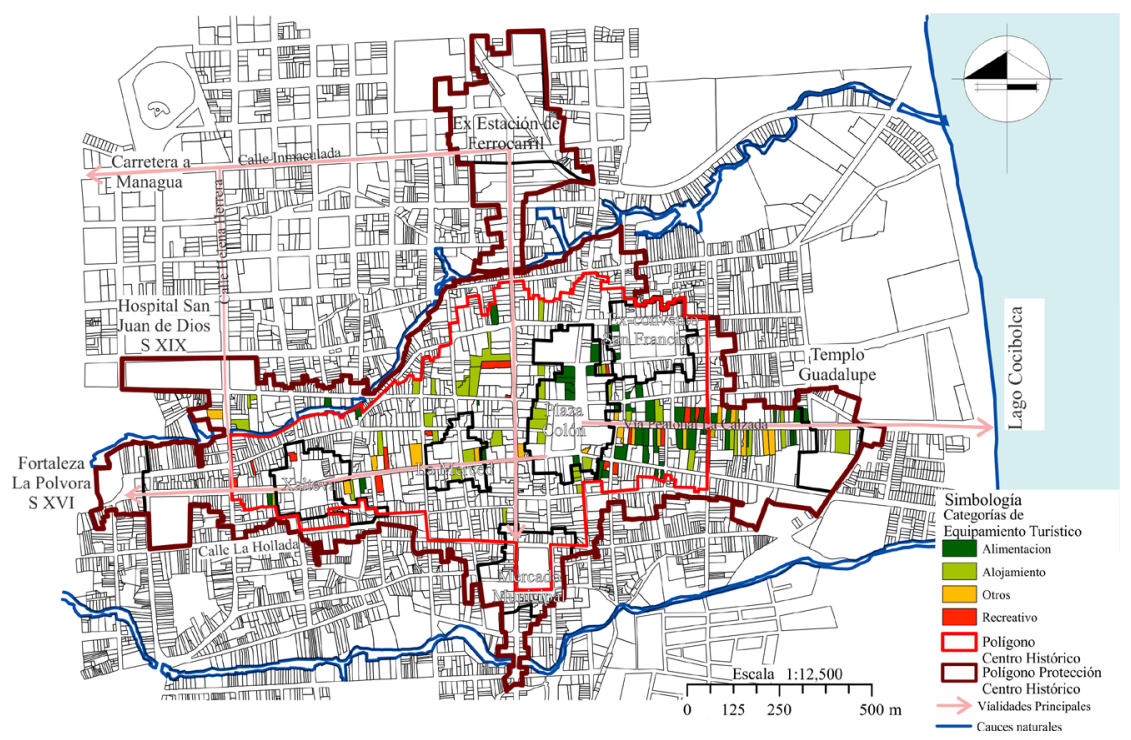

Nota. Figura del autor

La proporción de equipamiento turístico encontrado en el centro histórico de la ciudad Granada corresponde a $23.81 \%$ edificios (121 entre 508 edificios de la muestra). Esto confirma la existencia del fenómeno de la dotación de equipamiento turístico. Con base en los criterios anteriores, la muestra se redujo a 24 (19.8\%) edificios adaptados en el centro histórico de Granada. Luego se contabilizaron los espacios de estos edificios (125 espacios), y se realizó la caracterización previa a la dotación turística y otra al momento de tomar los datos. Se realizaron algunas medidas de algunos edificios donde fue permitido el acceso y registro de datos.

Se realizaron análisis probabilísticos para conocer qué tan significativos son los resultados obtenidos. La diferencia significativa entre frecuencia fue realizada por medio de análisis noparamétrico de ajuste de bondad de Ji-cuadrados. La relación nominal entre los valores, fue evaluada por medio de pruebas de Ji-cuadradas de contingencia. Las correlaciones nominales fueron medidas por medio del Coeficiente Corregido de Pearson (Cc) (Grande y Abascal, 2005, p. 132). Para el caso de datos paramétrico, se realizó un análisis de correlación de coeficiente $\mathrm{R}$ de Pearson. Todos estos métodos probabilísticos utilizados en el análisis, en la actualidad son considerados de uso común, razón por lo cual no se explica su mecanismo. Estos pueden ser consultados de manera detallada en Lind et al. (2008/2015), Triola (2009), Ritchie (2006/2008) y Kazmier (1978/1985).

A partir de la hipótesis planteada, se formularon las siguientes preguntas: (a) ¿existe alguna relación entre el cambio de uso de los espacios y la adaptación formal de los espacios arquitectónicos?, (b) ¿se producen un mayor número de adaptaciones formales a medida que existe un mayor número de cambios de usos en los espacios de cada edificio?, (c) ¿la similitud o diferencia en el uso de los espacios cuando se produce la dotación turística, influye en la forma de adaptar los espacios?, (d) ¿Cuál es el tipo de adaptación formal predominante?, (e) ¿Qué categoría de equipamiento turístico tiene relación con la variación de cambios de usos de los espacios de los edificios?

Con base en estas cinco preguntas se realizaron los siguientes análisis. El primero consistió en conocer la relación entre el cambio de uso y el cambio de forma a partir de frecuencias observadas. Las variables de análisis fueron la existencia de cambio de uso y la existencia de adaptación formal, 
ambos de tipo nominal, que podían tomar valores de "Si" o "No". La prueba probabilística aplicada fue del tipo Ji-cuadrada de contingencia. El tamaño de la muestra es de $n=125$, representada por los espacios de los 24 edificios. La Ho planteada es que no existe relación entre el cambio de uso y el adaptación formal de los espacios. La Hi es que si existe relación entre estas dos variables. Para un grado de libertad $(\mathrm{gl}=1)$ y un nivel de significancia de 0.05 , el criterio de rechazo de la Ho, consiste en encontrar valor de probabilidad de Ji-cuadrada, igual o superior $\chi^{2} \geq 3.84$.

El segundo análisis consistió en conocer la relación entre la cantidad de cambios de usos y la cantidad de adaptaciones formales. Las variables adoptaron valores escales de intervalos, en función de la cantidad de observaciones encontradas. La prueba probabilística realizada fue de correlación de Pearson (R). El tamaño de la muestra es de $n=24$, representada por los edificios registrados. La Ho planteada es que no existe relación donde a mayor cantidad de cambios de uso y existe mayor cantidad de adaptación formal de los espacios en los edificios. La Hi es que si existe relación entre estas dos variables. De forma empírica se afirma la existencia de correlación si se cumple el criterio de $(-0.5>r>0.5)$ (Lind et al., 2008/2015). Pero esto sólo es válido cuando existen un rango de 7-9 datos (Triola, 2013). Para conocer la significación del valor de R, se procedió a calcular la función de probabilidad t de Student, para afirmar la existencia de correlación en función de la cantidad de grados de libertad. La Ho es que el valor de $\mathrm{R}$ no es significativo y es casual, la Hi indica que el valor de R si es significativo. El criterio de rechazo de la Ho, con un nivel de confianza de 95\%, para $\mathrm{gl}=(\mathrm{n}-2)=22$, es que se cumpla la condición donde $\mathrm{t}$ sea menor que -2.074 o mayor que 2.074 $(2.074<\mathrm{t}<-2.074)$ (Lind et al., 2008/2015).

El tercer análisis realizado buscó establecer la relación entre las adaptaciones formales y el grado de similitud de uso del espacio, comparando el uso previo y uso actual. La variable de adaptación formal es tipo dicotómico nominal, en función de si existe o no cambio de forma ("Sí" o "No"). La variable que representa la similitud de usos previos y actuales toma los siguientes valores nominales: "Igual uso", "Uso similar" o "Uso diferente". El tipo de estadístico de prueba aplicado para la correlación entre estas dos variables fue la prueba de Ji-cuadrados de contingencia. El tamaño de la muestra es de $n=125$, representada por los espacios de los 24 edificios. La Ho afirma que no existe relación entre las adaptaciones formales y el grado de similitud de uso del espacio, la Hi plantea que si existe relación significativa. El valor mínimo esperado, para rechazar la Ho, con $\mathrm{gl}=2$ y un nivel de significancia de 0.05 , es de $\chi^{2} \alpha=0.05=5.99$.

El cuarto análisis consistió en conocer el tipo de adaptación formal predominante. La variable analizada fue la categoría de adaptación formal, que podía tomar cualquiera de los siguientes valores: "adición de volumen", "división del espacio", "sustracción" y "unión de espacios". La prueba probabilística aplicada fue de ajuste de bondad, para conocer la diferencia significativa entre las frecuencias observadas. El tamaño de la muestra es de $n=79$, representada espacios que si se adaptaron. La Ho afirma que no existe diferencia significativa ente las frecuencias, la Hi plantea que, si existe un tipo de adaptación predominante. El valor mínimo esperado, para rechazar la Ho, para un nivel de significancia de 0.05 , con $\mathrm{gl}=3$, es de $\chi^{2} \alpha=0.05=7.81$.

El quinto análisis consistió en establecer la relación entre la categoría de equipamiento turístico y la similitud de cambios que tienden a presentarse. Las variables son del tipo nominal. Los valores adoptados por la variable categoría de equipamiento son "alojamiento", "alimentación", "recreación" y "otros" -cómo se describió al inicio de la sección-. Los valores de similitud de uso son los mismo utilizados en el tercer análisis. Debido a que la distribución de datos obtenidos no cumple con los criterios de observaciones mínimas encontradas en todas las categorías para un análisis de contingencia, no se realizó ninguna prueba probabilística. 
La interpretación de los datos fue realizada en función de los resultados de pruebas de hipótesis probabilística. Finalmente se elaboró una postura crítica donde se contrastan los elementos teóricos y los resultados de la prueba de hipótesis, donde se explica el porqué de los resultados obtenidos.

\section{Resultados}

El primer resultado que se puede destacar es que, se logró conocer con base en las observaciones directas realizadas en campo -recuperadas del registro del primer recorrido-, que la mayor frecuencia de equipamiento turístico corresponde a los alojamientos, con un total de $48(38.71 \%)$ observaciones. En segundo lugar, los equipamientos de tipo alimentación con un total 43 (34.68\%) observaciones. En tercer lugar, se encontró la categoría de otros con 17 (13.71\%) observaciones. Finalmente, pero con una ligera diferencia en comparación a la categoría anterior, los tipos recreativos, con una representación de 16 (12.90\%) (Tabla 1) observaciones.

Tabla 1. Clasificación de los equipamientos turísticos observados en el Centro Histórico de la Ciudad de Granada

\begin{tabular}{lll}
\hline Equipamiento turístico & $\mathbf{f}$ & $\boldsymbol{f r}$ \\
\hline Alimentación & 48 & $38.71 \%$ \\
Alojamiento & 43 & $34.68 \%$ \\
Recreativo & 16 & $12.90 \%$ \\
Otros & 17 & $13.71 \%$ \\
Total & 124 & $100.00 \%$ \\
\hline
\end{tabular}

Nota. Elaborada por el autor

\section{Adaptación formal por cambio de uso en los espacios}

La dotación de uso turístico en edificios habitacionales, también produce adaptaciones formales en los espacios. Por ejemplo, un corredor o una sala de estar de una casa se convierte en sala de espera o recepción de un hotel. Pero en otras ocasiones, los dormitorios de una casa de habitación, continúa siendo habitaciones de hotel. Por esta razón, se buscó conocer (a) si la adaptación formal espacial se encontraba con mayor frecuencia cuando había dotación turística y (b) si el cambio de uso espacial tiende a producir mayor cantidad de adaptaciones formales que conservación formal. A partir de los registros realizados en los edificios estudiados, se encontró que existe una mayor frecuencia $(63.2 \%)$ de espacios que experimentaron adaptaciones formales cuando existe dotación turística. Aunque se conoce de antemano, que cuando existe dotaciones de usos turísticos y en el espacio se produce cambio de uso, las adaptaciones formales pueden o no producirse. No obstante, los datos indican que la mayor parte de los espacios (71.69\%) -76 de 106- que experimentaron cambio de uso también se adaptaron formalmente (Ver Tabla 2).

Tabla 2. Relación entre la dotación de equipamiento con el cambio de uso y forma del espacio.

\begin{tabular}{llllll}
\hline \multirow{2}{*}{ Cambio de Uso } & \multicolumn{5}{c}{ Cambio de Forma } \\
\cline { 2 - 6 } & No & $\%$ & Si & $\%$ & Total \\
\hline No & 16 & $12.80 \%$ & $01^{4}$ & $0.80 \%$ & 17 \\
\hline Si & 30 & $24 \%$ & $\mathbf{7 8}$ & $\mathbf{6 2 . 4 0 \%}$ & 108 \\
\hline Total & 46 & $36.80 \%$ & 79 & $63.20 \%$ & 125 \\
\hline \multicolumn{5}{c}{ Nota. Elaborada por el autor }
\end{tabular}

4 Debido a que una de las observaciones tiene un valor menor a 5, se aplicó la corrección de Yates. 
Con estos datos, se procedió a realizar una prueba de ji-cuadrados de contingencia . Como resultado se encontró que se cumple con la condición de rechazo de la Ho $\left(\chi 2=25.015>\chi^{2} \alpha=0.05\right.$ $=3.84)$ con un valor de $\mathrm{p}=5.69 \mathrm{e}-07$. Se encontró una relación significativa $(\mathrm{Cc}=57.74 \%)$ entre los cambios de usos de los espacios y la adaptación de la forma de los espacios. Esto permite afirmar que los cambios de usos inciden en la adaptación formal de los edificios con dotación turística.

\section{ificios}

Cantidad de adaptaciones formales en función de la cantidad de cambios de usos por ed-

Otra forma de poder identificar el impacto que pueden tener las dotaciones turísticas en las adaptaciones formales, es conociendo si al producirse la dotación, existe mayor número de adaptaciones formales a medida que existe mayor número de cambios de usos en los espacios, en cada edificio. Por esta razón, se indagó si existe alguna tendencia identificable, en donde se presenta mayor cantidad de cambio de uso de los espacios acompañados de una mayor cantidad de adaptaciones formales. Para esto se recurrió a un análisis de correlaciones. En los 26 edificios analizados, se representaron las frecuencias de cambio de uso observadas -eje x- y las frecuencias de adaptaciones formales -eje y- de cada edificio. Estos resultados se presentan en el diagrama de dispersión en la Figura 3.

Figura 3. Relación entre cambio de uso del espacio y cambio de forma.

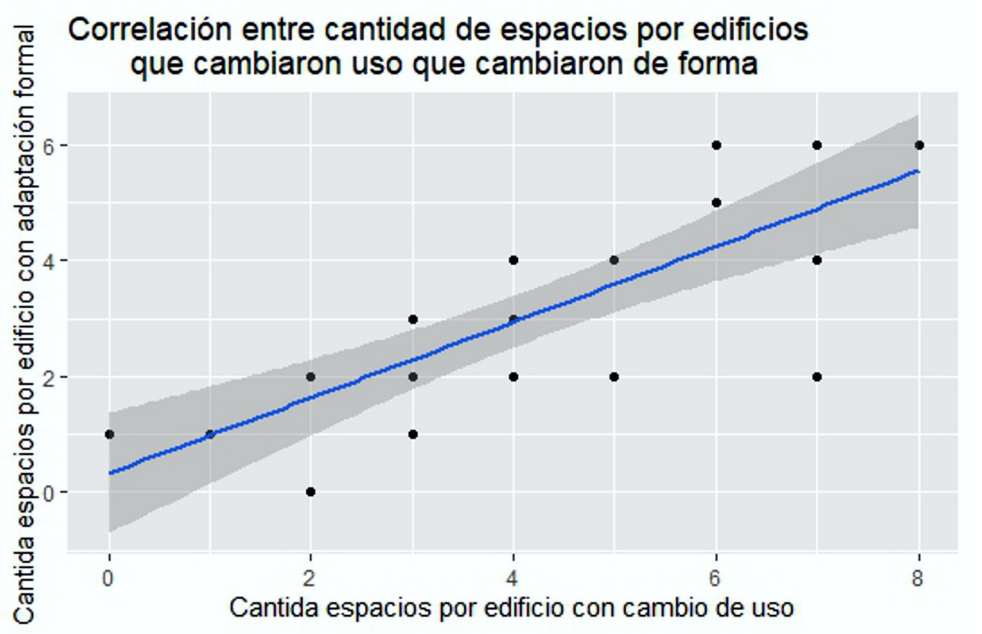

Nota. Elaborada por el autor

Se encontró una relación directamente proporcional, es decir que al aumenta el número de cambios de uso, también aumentan las adaptaciones formales. La correlación encontrada fue $\mathrm{R}=$ $77.21 \%$, con una determinación de $\mathrm{R} 2=59.62 \%$. El valor para la distribución $\mathrm{F}=3.44$, con $\mathrm{gl}=$ 24 , obtuvo un valor $\mathrm{p}=3.822 \mathrm{e}-06$, lo que indica un nivel de confianza de $99 \%$. En la prueba de importancia de la correlación t-student, se encontró un valor $\mathrm{t}=5.698$, para $\mathrm{gl}=22$, que permite rechazar la Ho, y afirmar que la correlación no es casual.

\section{Similitud de usos y cambio de forma}

Al analizar la relación entre las adaptaciones formales y el grado de similitud de uso del espacio, comparando el uso previo y uso actual, los resultados indican que la mayor frecuencia de cambios formales se produce cuando el uso actual del espacio es diferente al uso anterior. Esto se encontró en un total de 61 espacios $(75.30 \%)$ observados. Cuando el uso del espacio actual es similar al uso anterior, la modificación formal se reduce, encontrándose un total de 19 (23.45\%). Y cuando el uso 
del espacio actual es igual al uso anterior, los cambios en la forma son casi nulos, encontrándose solo un espacio (1.25\%). Estos datos se presentan gráficamente en la Figura 4.

Figura 4. Relación entre el grado de similitud del uso y el cambio deforma

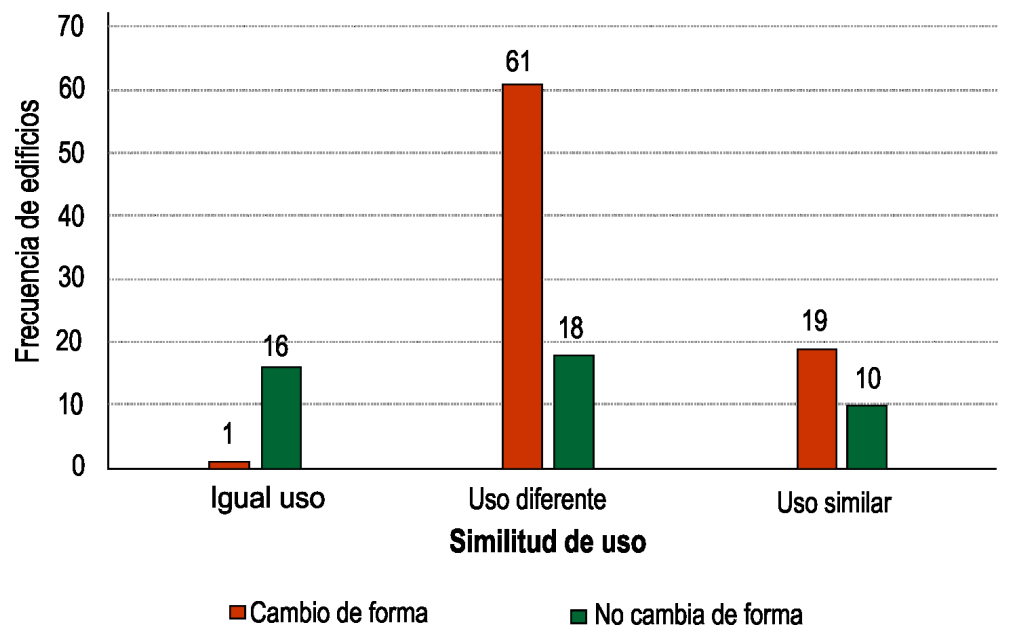

Nota. Elaborada por el autor

Los resultados de la prueba nominal indican que se cumple el criterio de rechazo de la Ho, $(\chi 2=$ $\left.31.217>\chi^{2} \alpha=0.05=5.99\right)$, con un valor de $\mathrm{p}=1.665 \mathrm{e}-07$ y que existe una relación $(\mathrm{Cc}=63.21 \%$ ) entre el grado de similitud del uso del espacio y las modificaciones generadas. Este resultado permite afirmar que, a mayor diferencia entre el uso previo con el uso actual en los espacios, existe mayor probabilidad de presentarse adaptaciones formales espaciales.

\section{El tipo de adaptación formal predominante}

El tipo de transformación que ocurre con más frecuencia es la división del espacio encontrada en 48 espacios (60.76\%), seguido por la adición de volumen en 26 espacios (32.91\%). En menor frecuencia se encuentran, la unión de espacios, con tres observaciones $(3.80 \%)$ y finalmente la sustracción con dos observaciones $(2.53 \%)^{5}$ (Ver Figura 5). La prueba probabilística aplicada fue de ajuste de bondad, para conocer la diferencia significativa entre las frecuencias observadas. Los resultados indican que la diferencia de los datos es significativa, y se cumple el criterio de rechazo de la Ho $\left(\chi^{2}=79.36\right)>\left(\chi^{2} \alpha=0.05=7.815\right)$.

Figura 5. Tipos de transformaciones en los espacios

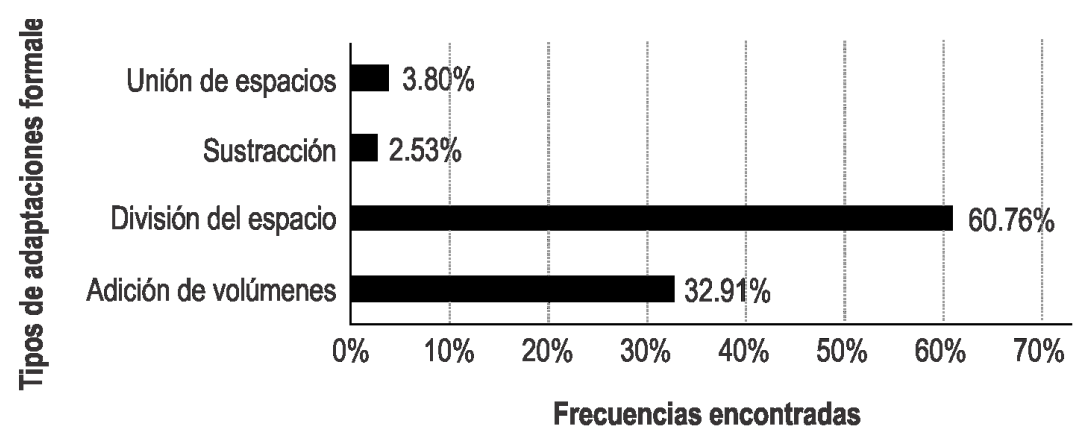

Nota. Elaborada por el autor

5 No se incluyeron las 46 observaciones, donde no existe cambio de forma, dato que se presentó en la sección de resultados de "Adaptación formal por cambio de uso en los espacios". 


\section{Cambios de uso según la categoría de equipamiento}

Ahora se procede a evaluar qué tipos de equipamientos turísticos tienden a generar cambios de usos en los espacios de los edificios. Para esto se relacionó la similitud de uso con el tipo de equipamiento. Se encontró que los edificios de alojamiento son los que presentan con mayor frecuencia cambios de usos en los espacios -uso diferente- (52 espacios, 41.60\%). Estos resultados se presentan gráficamente en la Figura 6.

Figura 6. Relación entre la categoría de uso Turístico y compatibilidad por uso de los espacios

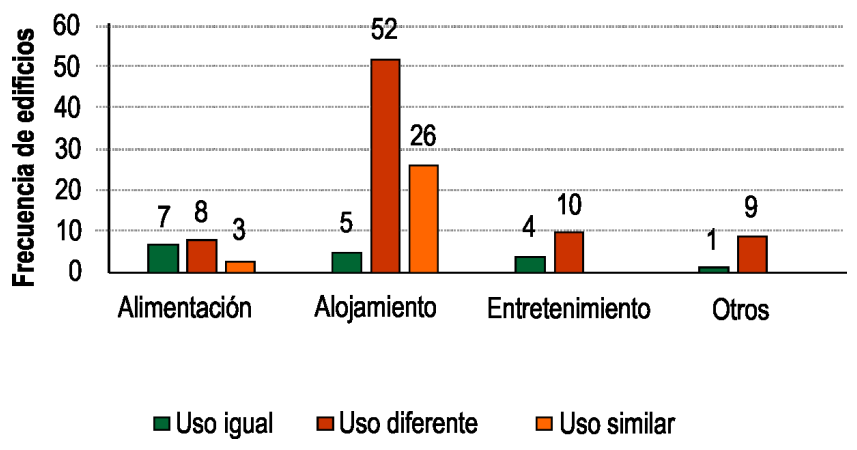

Nota. Elaborada por el autor

\section{Conclusiones}

En el desarrollo de este artículo se logró comprobar -con base en el caso del centro histórico de Granada, Nicaragua- que la dotación de equipamiento turístico en edificios habitacionales, tiende a generar cambio de uso de los espacios donde se realizan actividades turísticas, lo que genera con mayor frecuencia adaptación formal espacial. Se encontró la correlación de $77.21 \%$ que indica que, a mayor cantidad de cambios de usos en un edificio, se presenta mayor cantidad de modificaciones formales, con una determinación del 59.62\%.

Se logró comprobar que, (a) la adaptación formal ocurre con mayor frecuencia cuando el uso actual es diferente al uso previo; (b) que si el uso actual es similar al uso previo los cambios serán menos probables y dependerán de la manera en que estos se logren adaptar; y finalmente, (c) que si el uso actual es igual al uso previo los cambios se aproximan a cero. Esto confirma las aseveraciones planteadas por Flores (1999, p. 57), así como las recomendaciones de uso propuestas por organizaciones internacionales, tales como las indicadas por ICOMOS, para la conservación del patrimonio construido.

Los dos tipos de modificaciones formales con mayor frecuencia, la adición de volúmenes y la división del espacio, se encuentran asociadas con el cambio de usos. Se encontró que la mayor parte de las modificaciones formales son del tipo de División de los espacios y del tipo Adición de volumen, lo que tiene relación con los mencionado por Norberg-Schulz (2001, p. 74), en relación a los rangos máximos y mínimos para poder realizar actividades de manera confortable.

También se logró conocer que la mayor cantidad de cambios de usos fueron del tipo alojamiento. Al asociar estos dos resultados, se encontró que el predominio del tipo de modificaciones por división de los espacios, se debe a la dotación de equipamiento de alojamiento hotelero, quienes tienden a dividir los interiores de las habitaciones para añadir cuartos de baño. Así mismo, la adición de los espacios constituye la ampliación de la oferta de servicios turísticos. Esto genera cambios en la configuración, debido a que al menos en uno de los espacios previamente existente 
experimentaron una adición, sustracción o división de espacios que altera la configuración previa del edificio.

Dependiendo de las necesidades a desarrollarse en un espacio existente y las formas de los espacios, surgirán los modos de ocupación de los espacios. Y en la medida en que la necesidad de uso se adapte a la forma espacial se logrará un espacio funcional. Las adaptaciones están relacionadas con los modos de ocupación del espacio y son necesarios para la existencia del edificio. Son producidas por el hombre, para el uso del espacio y es parte de la adaptación en el medio. La necesidad de transformar existe en el ser humano mientras conserve los rasgos fundamentales de su estructura biológica, psicológica y social contemporánea (Saldarriaga, 1981, pp. 15-16). La prevalencia de la ciudad y la arquitectura dependen de estas transformaciones. Se trasforma para adaptarse nuevas condiciones. Por tanto, las transformaciones están comprendidas dentro un proceso natural en el desarrollo.

Se encontró, con base en los datos registrados, que los edificios habitacionales al cambiar de uso también cambian sus espacios, según las actividades que se desarrollen en el tipo de equipamiento turístico. Estas formas de ocupar el espacio siguen las siguientes tendencias. Cuando los corredores son lo suficientemente anchos para añadir otro espacio, estos son ocupados por los hoteles para la adición de habitaciones. Los restaurantes ocupan los corredores como áreas de mesas y ubicar la cocina. Los dormitorios de las viviendas son ocupados como habitaciones de hotel, subdividiéndose el espacio con la adición de un cuarto de baño. Los museos ocupan todos los dormitorios como espacios de exhibición. Los bares y restaurantes, pueden o no utilizar los dormitorios. Cuando los utilizan los convierten en almacén.

Los espacios localizados en la parte frontal del edificio, son ocupados como recepción o venta de alimentos y bebidas por los hoteles; como área de venta en las tiendas de souvenir y áreas de mesas por los bares. Los patios que son modificados, comúnmente utilizados por los hoteles para aumentar la cantidad de habitaciones con la adición de un volumen, y donde se les permite, construyen piscinas (en el caso particular de Granada el segundo patio). Cuando los edificios habitacionales solo tienen un patio, este es adaptado.

Entre las limitaciones encontradas en el desarrollo de la investigación, se puede mencionar el acceso a los edificios, así como la carencia de información previa al uso turístico. La primera limitante, está relacionada con el hecho que en este tipo de edificios se ofertan servicios y cualquier visitante que no contribuye a generar ganancias, no es de interés para los locatarios. La segunda razón podría estar relacionada con las implicaciones de las adaptaciones en los edificios patrimoniales, ya que en su mayoría están prohibidas o limitadas. En cuanto, a la carencia de registros previos al uso turístico lamentablemente no se puede hacer mucho. La posible solución está en consultar los registros de los habitantes, historiadores locales o arquitectos que hayan intervenido edificios, esperando que conserven dichos datos.

Queda pendiente investigar si estos mismos patrones se repiten en otros centros históricos. Si existen algunas variantes en las adaptaciones, con tendencia a la conservación, a partir de los reglamentos y normas vigentes en otros centros históricos. Si al aumentar el número de observaciones, es posible tener una conclusión probabilística contundente sobre si existe un tipo de equipamiento turístico que tienda a generar mayor número de adaptaciones. Quizás alguno de estos problemas pueda interesar a algún lector en continuar esta línea de investigación, para responder estas u otras preguntas relacionadas. 


\section{Bibliografía}

Ashworth, G. J., \& Tumbridge, J. E. (2000). The Tourist-Historic City: Retrospect and Prospect of Managing the Heritage City. Amsterdam: Oxford.

Bazant S., J. (2005). Manual de Diseño Urbano. México, D. F.: Trillas.

Boullón, R. (1985). Planificación del espacio turístico. México D.F.: Trillas.

Camacho Cardona, M. (1993/2007). Diccionario de Arquitectura y Urbanismo. México: Trillas.

Carter, H. (1972/1974). El estudio de la geografía urbana. Barcelona: Edición Española del Instituto de Estudios de Administración Local.

De la Torre Padilla, O. (1997). El turismo: fenómeno Social. México D.F.: Fondo de Cultura Económica.

Eco, U. (1974/1986). La Estructura Ausente. Barcelona: Editorial Lumen.

Ellisa, E., Narumi, K., \& Tahara, N. (1997). A study on the building transformation in the historical Core City Area. J.Archit. Plann.Enviren.Eng(501), 163-173. https://www.jstage.jst.go.jp/article/aija/62/501/62_KJ00004222332/_pdf

Eyüce, Ö., \& Eyüce, A. (2010). DESIGN EDUCATION FOR ADAPTIVE REUSE. International Journal of Architectural Research, 4(2-3), 419-428.

Flores Arias, V. (1999). Aspectos teóricos y prácticos sobre la reutilización de los espacios construidos. Anuario de Estudios de Arquitectura. Historia, crítica y conservación, 53-73.

Grande, I., \& Abascal, E. (2005). Análisis de encuestas. Madrid: ESIC Editorial.

Groat, L., \& Wang, D. (2012). Architectural research method. New Jersey: Wiley.

Hernández Díaz, E. A. (1991). Planificación Turística, un enfoque metodológico. México D.F.: Trillas.

Kazmier, L. J. (1978/1985). Estadísticas Aplicadas a la Administración y a la Economía. (C. Esguerra, \& C. De Barella, Trads.) México, D.F.: McGraw Hill.

Köpsel, V. (2019). Climate Adaptation, People-Place Relationships, and Local Actors: Reviewing the Literature. In New Spaces for Climate Change. (pp. 23-34). RaumFragen: Stadt - Region - Landschaft: Springer VS, Wiesbaden. doi:10.1007/978-3-658-23313-6_2

Lind, D., Marchal, W., \& \& Wathen, S. (2008/2015). Estadística Aplicada a los Negocios y la Economía (16 ed ed.). (M. D. Obón León, \& J. León Cardenas, Trads.) México, D.F: McGraw Hill.

Mattos, C. A. (2002). Transformación de las ciudades latinoamericanas. ¿Impactos de la globalización? EURE, 28(85). http://dx.doi.org/10.4067/S0250-71612002008500001

Norberg-Schulz, C. (1967/2008). Intensiones en Arquitectura (3ra ed.). Barcelona: Gustavo Gili.

Oficina de Preservación del Centro Histórico de Granada Nicaragua. (2002). Resumen ejecutivo, Plan de revitalización del Centro Histórico de Granada. Granada, Nicaragua: Resumen ejecutivo, Plan de revitalización del Centro Histórico de Granada. 
Oficina de preservación y conservación del centro histórico de Granada. (1999). Granada: Historia y desarrollo. Granada: Oficina de preservación del Centro Histórico de Granada.

Oliver, P. (2006). Built to Meet Needs: Cultural Issuues in Vernacular Architecture. Oxford: Elsevier.

Reyes, M. A. (2002/2004). Catálogo de bienes patrimoniales Granada, Nicaragua. (coord.). $\therefore$ Granada: Oficina del Centro Histórico /Alcaldía de Granada / Agencia Española de Cooperación Internacional para el Desarrollo (AECID).

Ritchie, F. J. (2006/2008). Estadísticas para ciencias sociales (2da ed ed.). (J. H. Romo Muñoz, J. Yesca Milanes, J. León Cárdenas, \& J. Velázquez Arellano, Trads.) México: Mc Graw Hill.

Robinson, P. (2012). Tourism: The Key Concepts. London: Routledge.

Saldarriaga Roa, A. (1976/1981). Habitabilidad. Bogotá: Escala.

Soewarno, N., \& Duhita, D. (2019). The Transformation of Heritage Buildings as Tourist Attraction: Adaptive Re-use of Colonial Buildings. Advances in Engineering Research(156), 131-140. doi:https://dx.doi.org/10.2991/senvar-18.2019.19

Specht, J. (2014). Architectural Tourism. Building for Urban Travel Destinations. Wiesbaden, Germany: Springer Gabler. https://doi.org/10.1007/978-3-658-06024-4

Suebsuk, N., \& Nakagawa, O. (2015). Transformation and Modification of Historical Building Use, and Owner Motivation for Urban Conservation in the Nineizaka and Sanneizaka Preservation District, Kyoto, Japan. Athens Journal of Tourism, 2(1), 37-54. doi:https://doi.org/10.30958/ ajt.2-1-3

Triola, M. F. (2013). Estadística. México: Pearson.

UNESCO/PNUD. (1977). Carta de Quito. Coloquio sobre la conservación de los Centros Históricos Ante el Crecimiento de las Ciudades Contemporaneas (pág. 13). Quito: UNESCO. 